UDK 78:930

Arnold Feil

Tübingen; Korr. Mitglied der Slowenischen Akademie der Wissenschaften und der Künste

Dopisni član Slovenske akademije znanosti in umetnosti

\title{
Musik als Geschichte
}

\section{Glasba kot zgodovina}

Ključne besede: zgodovina glasbe, predmet glasbene zgodovine; notacija, ustno izročilo, ideja napredka

\section{IZVLEČEK}

Članek skuša razjasniti dileme, ki se pojavljajo ob pisanju zgodovine glasbe. Tako se avtor dotakne vprašanja, kaj so to dejstva glasbene zgodovine in njihova interpretacija, skuša določiti predmet glasbene zgodovine, uvaja pomembno razliko med "ustnimi“ in "pisnimi" glasbenimi kulturami ter se sprašuje, ali je zgodovinski lok mogoče opazovati skozi optiko stalnega napredka. Poseben problem predstavlja tudi periodizacija glasbene zgodovine, ki pa ima v luči vprašanja, kaj je glasba, šele sekundarni pomen.
Keywords: history of music, the object of the history of music; notation, oral tradition, idea of progress

\section{ABSTRACT}

The article tries to clarify the dilemmas which arise when writing a history of music. The author raises the question abut the facts of music history and their interpretation, he tries to determine the object of history of music, introduces the important distinction between "oral" and "notational" music cultures and finaly examines the possibility of understanding the historical arch in light of idea of progress. Special problem represents the periodisation, which has next to the question what is music secondary value. 


\section{Fakten der Musikgeschichte}

Mehr als sonst in "Musikgeschichten" ist in dem Buche, aus dem der folgende Text zitiert ist (METZLER MUSIK CHRONIK vom frühen Mittelalter bis zur Gegenwart von Arnold Feil, Verlag J. B. Metzler Stuttgart, Weimar, 1993; 2., erweiterte Auflage 2005), die Darstellung auf Fakten gestützt, und zwar, wenn möglich, in deren einfacher zeitlicher Folge. Aber was sind denn die Fakten der Musikgeschichte? Die Jahreszahlen jedenfalls zeugen nur von ihnen; die Fakten sind in gewisser Weise aus den Daten rekonstruiert. Dass solche Rekonstruktion immer zugleich und notwendig Interpretation ist, mit der der Historiker sein und seiner Zeit Interesse an der Geschichte, an der "Sache", einbringt, muss man einsehen und akzeptieren. Für eine "normale" Darstellungsweise der Musikgeschichte bietet sich das Modell der Biographik an, "die ,lebensgeschichtliche' Metaphorik, zu der die Historiker der Kunst seit Winckelmann und Herder unwillkürlich immer wieder greifen, weil die ,Lebensalter' einer musikalischen Gattung oder eines Stils als Analoga zu denen einer Person aufgefasst werden können, wodurch die erzählte Geschichte Struktur und festen Umriss erhält" (Dahlhaus 1977). Solche Struktur ist indessen so oft als die der Sache selbst angesehen und dargestellt und die Relevanz der Darstellung mit der des Historischen verwechselt worden, dass ein allgemeines Misstrauen gegen das Selbstverständliche traditioneller Geschichtsschreibung Platz gegriffen hat. Man möchte keine "Darstellung" mehr lesen, man möchte, dass die "Dokumente" selbst reden, - und man möchte glauben, die Chronologie spreche schon ihre eigene Sprache. Dokumentensammlungen und chronologische Werkverzeichnisse sind deshalb in der Musikwissenschaft oft an die Stelle repräsentativer Musikerbiographien getreten, und die Musikgeschichtsschreibung im engeren Sinne liegt im Argen. Die Musikwissenschaft hat ihre philologische, das heißt textinterpretierende und geschichtlich darstellende Aufgabe vernachlässigt und sich weitgehend auf die nur auch zur Philologie gehörende Aufgabe des Sammelns von Quellen, der "Herstellung" von "Texten" und auf die Chronologie (und die Detailforschung dazu) zurückgezogen. Dass solche Musikwissenschaft schon "Musikgeschichte" ergebe oder gar sei, ist derselbe Aberglaube wie der, dass Quellensammlungen einen Wert an sich hätten und Dokumente und Chronologie allein etwas aussagten, nämlich über die Musikgeschichte, für die sie, wie gesagt, doch nur Quellen sind. Trotzdem bleibt ein Unbehagen gegenüber Darstellungen sowohl der Musikgeschichte als auch von "Leben und Werk" der großen Meister - es sei denn, sie würden enger und leichter durchschaubar an die Dokumente und Daten geknüpft.

Indessen, wenn auch die Darstellung der Fakten eng an die Dokumente und die Daten gebunden bleibt, was sind - man muss die Frage wiederholen - die Fakten der Musikgeschichte? Die Lebensdaten der Musiker, die Entstehungs- und Aufführungsdaten von Werken, das alles hat seine eigene, durchaus faktische Bedeutung jedenfalls erst in zweiter Linie; in erster Linie zeugt es, muss es zeugen von und für Musik. Sie aber, die Musik, ist der erste - früher hätte man gesagt: der vornehmste - Gegenstand der Musikgeschichte. Doch ist Musik überhaupt ein "Gegenstand"? Man sieht, die Frage nach dem Objekt der Geschichte stellt sich dem Musikhistoriker schärfer als jedem anderen Historiker; sie muss deshalb zuerst überlegt werden. Sodann gilt es, aufgrund der Besonderheit des "Gegenstandes Musik" gewisse Besonderheiten auch der Musikgeschichtsschreibung zu erörtern.

Festzuhalten ist jedenfalls dies: Eine adäquate Darstellung der Musikgeschichte muss zuerst und vor allem dahin führen, dass wir, die wir alle hören und Erfahrungen mit Musik und musikalischen Kunstwerken haben, wieder neu lernen, uns zum musikalischen Kunstwerk richtig zu verhalten, nämlich so, dass das, was den registrier- und fixierbaren Daten und Fakten ihren Daseinssinn verleiht, überhaupt zur Wirkung kommt als Musik. Dass die Musik und ihre Wirklichkeit dann auch zur Sprache kommen, ist sekundär, doch kann das Zur-Sprache-Bringen wiederum den Lernprozess befördern. 


\section{2. Über die Wirklichkeit von Musik}

Musik muss, um Musik zu sein, erklingen. Erklingen ist an Gegenwart geknüpft. Musik verklingt wie Gegenwart verrinnt. Wie kann dann Musik Geschichte haben - nicht nur haben, sondern sein? Die Antwort ist schwierig und einfach zugleich: in der Gegenwart, in der sie erklingt, das heißt in stattfindender Vergegenwärtigung. Der Musiker macht, der Hörer hört Musik, auch der Vergangenheit, nur als gegenwärtige Musik. Wie, muss man dann fragen, ist Musik der Vergangenheit auf uns gekommen? In Kompositionen, das heißt in notenschriftlich niedergelegten Anweisungen zum Musik-Machen. Denn die Komposition ist für den Ausführenden im Grunde nichts anderes als die Aufforderung, das Notierte, das als solches nicht schon die musikalische Wirklichkeit sondern erst ihre Voraussetzung ist, ins Erklingen zu überführen; die Komposition verlangt den letzten, beim Komponieren intendierten Schritt auf dem Wege des Entstehens von Musik als Schritt zur Verwirklichung bei der Aufführung, das heißt im musikalischen Vortrag: Vergangenheit und Gegenwart müssen eine einzige Wirklichkeit werden und sein, wenn Musik verantwortungsvoll gemacht wird und erklingt und wahrgenommen wird, gleichgültig ob die Akte des Machens und Hörens, der Produktion (nicht der Re-Produktion, wie man fälschlich sagt) und der Rezeption bewusst vollzogen werden oder unbewusst.

Musikkulturen, die keine Notenschrift und keine Komposition kennen - und nur die europäischabendländische Kultur hat für ihre Kunstmusik eine Notenschrift entwickelt, die zur Komposition dient -, kennen dieses Interpretationsproblem so nicht, sie haben das Problem alte Musik/neue Musik, Musikgeschichte/Gegenwart der Musik nicht, ja sie haben in gewisser Weise überhaupt keine Musikgeschichte. weil all ihre Musik nur gegenwärtig existiert, weil sie im Moment des ZumErklingen-Kommens jeweils und immer wieder neu geschöpft wird, und zwar aus einem Repertoire, für das das Bewusstsein keine historisch triftige Scheidung in alt oder neu kennt. Eine "Geschichte der abendländischen Musik" hat also nicht nur im Denken von Geschichte und Geschichtlichkeit sondern im Gegenstand selbst prinzipiell andere Voraussetzungen, als sie jede "Musikgeschichte" einer anderen Kultur hätte.

Selbst für die europäisch-abendländische Kunstmusik galt, obwohl sie seit dem 12./13. Jahrhundert mit Notenschrift und Komposition Denkmäler ihrer Geschichte setzen konnte und gesetzt hat, bis zur großen Wende ins historische Zeitalter um 1800, dass eine jede Musik die vorhergehende verdrängt hat, und dass für den Musiker die Aufgabe des Musikmachens in aller Regel und selbstverständlich in Komposition und Aufführung neuer und eigener Werke bestand. Das musikalische Bewusstsein war also jeweils bestimmt von der Musik der eigenen Zeit, und zwar ausschließlich. Ungefährdet von aller Vergangenheit als geschichtlicher Realität war Musik zu definieren, war über Musik zu philosophieren; man konnte allein aus der eigenen Erfahrung wissen, was das ist: Musik. Seit aber Händels und danach Haydns Oratorien, seit vor allem Beethovens Sinfonien nicht mehr vergingen und durch die Werke ihrer Schüler und Nachfolger nicht mehr verdrängt worden sind, seitdem ist das anders, seitdem ist ein völlig neuer Zustand von Musik eingetreten. Von nun an kann Musik nicht mehr nur sein, was in der jeweiligen Gegenwart entsteht und erklingt. Musik ist vielmehr auch all das, was verklungen ist, aber wieder neu zum Erklingen gebracht werden will, weil das Bewusstsein von Musik jetzt alle Musik begreift. Jetzt hat die Definition von Musik alles einzuschließen, was Musik war und ist, in Geschichte und Gegenwart, innerhalb und außerhalb der eigenen Musikkultur.

Als Felix Mendelssohn Bartholde am 11. März 1829 in Berlin mit der 1791 gegründeten "Singakademie "J. S. Bachs Matthäuspassion aufgeführt, nämlich der Vergangenheit entrissen und 100 Jahre nach ihrer ersten Aufführung als aktuelle Musik zu einer neuen Wirklichkeit und Wirksamkeit gebracht hat, war das neue Zeitalter der Musik endgültig angebrochen. Zugleich war damit aber auch, was für die europäische Kunstmusik realiter schon immer gegeben war, zum ersten Mal 
zum Problem geworden, nämlich die dreifache Daseinsform (Schering 1933) des musikalischen Kunstwerks, sofern es in Komposition schriftlich fixiert ist:

1. In Notenschrift ist das Werk unveränderlich fixiert, dem zeitlichen Verlauf enthoben, der sinnenden Betrachtung zugänglich.

2. Erklingend wird es veränderlich und muss es sein, fällt es der Zeitflucht und nach, ja mit jeder Aufführung wieder der Vergänglichkeit anheim, nur hier allerdings wird es sinnlich wahrnehmbar, wird es zur Wirklichkeit von Musik.

3. In unserem musikalischen Gedächtnis und Bewusstsein schließlich bleibt es haften als Eindruck (Abdruck) der klingenden Wirklichkeit, nun der zeitlichen Dimension wieder enthoben, in gewisser Weise frei von den Bedingungen seiner fixierten Komposition, dafür aber der Arbeit und Verarbeitung des verstehenden Denkens und des Geistes im allgemeinen überstellt.

Die Interpretation von Werken der Musik hat stets alle drei Daseinsformen zu bedenken, in gegenseitiger Spannung zu halten und in den einen Prozess des Interpretierens zu bringen, in den Prozess, der sich immerzu erneuern muss, will er Wirklichkeit schaffen.

All dies hat auch zu bedenken, wer "Musikgeschichte" schreibt, Geschichte von Musik, von Werken, die wir als musikalische Denkmäler in Noten vor uns liegen haben, Geschichte auch von musikalischen Ereignissen, die immer wieder unzählig viele Menschen in der Musik Möglichkeiten von Welt haben erfahren lassen, von Eindrücken auch im Gedächtnis derer, die Musik machen und hören. All dies zu bedenken hat aber ebenso der, der "Musikgeschichte" liest. Denn der Autor, der in verständlicher Weise über Musik schreiben will, muss beim Leser musikalische Erfahrung voraussetzen. Es kann nur mitgeteilt und verstanden werden, was vom Schreibenden wie vom Lesenden gesehen, gehört, erfasst - mit einem Wort: erfahren ist, sonst stehen Worte und Sätze im Leeren. Wenn ein Buch über Musikgeschichte, das ist: über die geschichtliche Wirklichkeit von Musik, konkret etwas aussagen soll, dann ist das nur möglich, wenn und soweit die Erfahrung des Lesers - sei es direkt, sei es indirekt - mit dieser Wirklichkeit in Fühlung steht. Der Leser wird also nicht umhin können, sich gegebenenfalls um eine Vorstellung von der Musik zu bemühen, zu deren Geschichte die Daten versammelt und zu einer "Musikgeschichte" ausgeschrieben sind.

\section{3. "Mündliche Musik" und "Schriftliche Musik"}

Zu bedenken ist sodann dies: Die Musik, von der hier berichtet wird, ist vom Gesamt der Musik, das den Menschen jeweils zur Verfügung steht, von aller Musik, die sie haben und machen, nur ein Bruchteil. Man geht, wenn man von Musik und ihrer Geschichte spricht, in der Regel selbstverständlich von der sogenannten Kunstmusik aus und behandelt die sogenannte Volksmusik bestenfalls am Rande und nur dort, wo sie von Einfluss auf die Kunstmusik ist. Diese Trennung in Volks- und Kunstmusik ist in Wirklichkeit die Unterscheidung von zwei Bereichen der Musik. Am ersten haben alle Menschen teil, am zweiten nur diejenigen, die eine besondere musikalische Bildung erhalten. Diese beiden Bereiche, die hier nach einem musiksoziologischen Gesichtspunkt getrennt sind, korrespondieren mit zweien, die im engeren Sinne musikalisch zu definieren sind. Der erste Bereich ist zu beschreiben als der, dessen Musik in mündlicher Überlieferung entsteht, lebt und tradiert wird (und die in der Regel nur dann schriftlich fixiert wird, wenn die Überlieferung gefährdet ist oder beeinflusst werden soll); zu ihm gehört vor allem die Volksmusik aber auch die einstimmige vokale Kunstmusik etwa der Trouvères und Trobadors, des Minne- und des Meistergesangs, zu diesem Bereich gehören aber auch - nicht zu vergessen! - ursprünglich der Gregorianische Choral und das Kirchenlied. Der zweite Bereich ist der der "schriftlichen Musik", das heißt der Komposition und damit der Polyphonie. 
Man mache sich das bewusst: Kinderlieder, Wanderlieder, Straßenlieder, überhaupt Volkslieder und Volksmusik und heutzutage Songs und Schlager, aber auch Kirchenlieder, lernt man nicht und singt und spielt man nicht nach Noten, sondern man hört sie von anderen und lernt sie hörend und singt und spielt sie dann nach, wenn man sie kennt und kann; und wer dieses oder jenes Stück auch schriftlich haben will, der kann sich aufschreiben, was er im Ohr hat (Notation als Nach-Schrift). Im Bereich der schriftlichen Musik hingegen entsteht jedes einzelne Stück aus seinen Noten (Notation als Vor-Schrift), indem man, was da schriftlich von einem Komponisten verfasst ist, abspielt oder absingt, wobei man nicht nur Noten lesen und nach Noten singen und spielen können sondern überhaupt im Bereich der Kunstmusik bewandert sein muss.

Diese für Musik und Musikleben grundlegende Tatsache der Verschiedenheit von Bereichen der Musik kann man sich auch an folgender Überlegung klarmachen. Dass die Kompositionen der Hofkapellmeister und Hofkomponisten im Wien des 18. Jahrhunderts nicht für die sogenannte Grundschicht des Volkes bestimmt waren, ist anzunehmen. Dass aber die Kaiserin Maria Theresia ihren vielen sehr geliebten Kindern keine Kinderlieder gesungen habe, ist höchst unwahrscheinlich. Und sollte sie wirklich nicht selbst Wiegen- und Kinderlieder gesungen und mit ihren Söhnen wenigstens hie und da "Hoppe, hoppe, Reiter" gespielt haben, so haben es die Ammen und Kindermädchen getan. Die kleinen Erzherzöge und Erzherzoginnen lernten sehr wohl "die lebendige Stimme des Volkes" (Herder), sie kannten sicherlich und konnten auch die Lieder des Volkes, die Sprüche und Abzählreime, die Jahreszeitenlieder und die Kirchenlieder, wenn nicht alle, so doch viele, wie alle Menschen des Volkes, zu dem auch sie gehörten. Denn nicht in erster Linie was das niedere Volk singt, macht das Volkslied aus - jedenfalls früher -, sondern was in mündlicher Überlieferung lebt und was lebendig ist in den "natürlichen" Bindungen, in Funktionen des Lebens. Das Volkslied entspricht in gewisser Hinsicht dem Dialekt der Sprache: es gehört zu jener Schicht des Analphabeten. die wir in uns haben und die in all dem Dialektund Umgangssprachlichen wie Reimen, Witzen, Spottversen, Schimpfwörtern und eben auch in Volksliedern nach außen dringt. Das für die europäische Musik grundlegend wichtige Gegensatzpaar lautet also nicht: Musik der Grundschicht und Musik der Oberschicht, sondern Musik in mündlicher Überlieferung (auch wenn sie auf Komposition zurückgehen sollte) und komponierte Musik. Komponierte Musik zu verstehen ist zwar einem Teil der Gesellschaft vorbehalten, der eine besondere musikalische Bildung genießt, die mündlich überlieferte Musik aber ist, zumindest in ihren wichtigeren Gattungen, Musik des ganzen Volkes und nicht nur einer seiner Schichten.

Trotzdem ist es sinnvoll, wenn der Musikhistoriker heute wie seit Forkel stets und immer wieder "die Musik der unteren Volksschichten" aus der Musikgeschichte ausklammert, weil sie zu dieser ebensowenig gehöre wie "das Handwerk der Tüncher zur Kunstgeschichte" (Allgemeine Geschichte der Musik, 1788-1801, Einleitung). Wer "Musikgeschichte" schreibt, schreibt ja doch Geschichte der musikalischen Kunst, das heißt der komponierten polyphonen, der an Schrift gebundenen und durch "musikalischen Satz" gekennzeichneten Musik (auch ihrer Ausführung und Lebensweise) und nicht Geschichte der in mündlicher Überlieferung lebenden einstimmigen oder lediglich volkstümlich-vielstimmigen Musik - wie könnte man auch die Geschichte einer Erscheinung schreiben, die jeweils nur in der Gegenwart existiert, weil sie nichts Bleibendes hat - es sei denn, das immer nur Gegenwärtige wäre das Bleibende - und deshalb keine Zeugnisse der Vergangenheit aufweisen kann. (Wenn man den Bereich mündlicher Musik hingegen als den einer "niederen Kunst" bezeichnet und deshalb nicht in die Betrachtung der Musikgeschichte einbezieht, so offenbart sich darin ein Irrtum.)

All das ist hier nur kurz, vielleicht gar verkürzt dargestellt, aber es muss wenigstens angedeutet werden, weil derjenige, der sich mit Musikgeschichte denkend befasst. das Umfassende der Musikkultur mitdenken muss, auch wenn er sich nur mit dem Teilgebiet der Kunstmusik befasst. 


\section{4. "Entwicklung in der Musikgeschichte?}

Wer Geschichte betrachtet, gerät leicht in die Gefahr, nichts als Entwicklungen zu sehen, vielleicht sogar im Ganzen der Geschichte ein Gerichtet-Sein zu vermuten oder auch zu entdecken. Die Gefahr ist umso größer, wenn aus älteren Epochen nichts mehr oder nur wenig überliefert ist, und diese Epochen zudem ferngerückt sind, während jüngere durch ihre Nähe und aus der Lebendigkeit einer noch wirksamen Anschauung ihrer Überlieferung gleichsam natürlicher Weise von größerer Bedeutung zu sein scheinen. Etwa mit dem vielberufenen "finsteren Mittelalter" (im Kontrast zum emanzipatorischen Aufbruch und Anspruch der Humanisten so benannt) haben wir für die Musik noch immer unsere Schwierigkeiten, obwohl wir wissen und einsehen könnten, dass eine Zeit wie die Kaiser Friedrichs II. (1220-1250), deren Kunst und Kultur wir zu bewundern gelernt haben, kaum mit einem Stand der mehrstimmigen Musik vorliebgenommen haben dürfte, der gemessen an der Gesamtkultur primitiv zu nennen wäre und oft genug genannt wird, und das, obwohl die Musik als eine der sieben ArTes LIBERALEs im Denken und im Bildungssystem jener Zeit bekanntlich von hoher Bedeutung gewesen ist. Wir kennen einfach die Musik des hohen Mittelalters nicht genügend und sind deshalb nur allzu schnell bereit, sie kurzerhand als noch nicht entwickelt zu beschreiben. Dieses "noch nicht" ist eine Gefahr für alle Geschichtsschreibung, ebenso das "schon", und wir sollten skeptisch sein gegenüber Feststellungen, die man allerorten lesen kann und die durchaus lauten können: "Schon im Mittelalter gab es instrumentale Tanzmusik." Hier wird an der Interpretation aus Nichtwissen infolge mangelnder Überlieferung mit einem Male deutlich, dass und wie wir Geschichte als Entwicklung und befangen im Fortschrittsglauben zu interpretieren geneigt sind. Dass alles Neue sich in gewisser Weise herausbildet und trotzdem oft genug wie aus dem Nichts zu entstehen scheint, ist damit nicht bestritten, wohl aber der Gedanke des Fortschritts in der Geschichte und erst recht der von Notwendigkeiten in - immer nur scheinbar - kausalen Zusammenhängen.

\section{Das Problem der Epochengliederung der Musikgeschichte}

Ein Problem besonderer Art, schwierig und nie befriedigend zu lösen, für eine "Misikgeschichte" indessen von besonderer Bedeutung, ist das der Epochengliederung der Musikgeschichte. Sicherlich ist es von geringerer Bedeutung, als man ihm gelegentlich zumisst, aber es ist keinesfalls nur ein Problem der Darstellung, sondern eines der Geschichte selbst und unseres Bewusstseins von der Geschichte.

Doch dieses letzte Problem sei hier nicht weiter erörtert; für die Frage „Was ist das, die Musik?» ist es, wie gesagt, ein nachgeordnetes, nicht das erste und wichtigste Problem - so schwierig es auch sein mag.

\section{POVZETEK}

Članek skuša razjasniti dileme, ki se pojavljajo ob pisanju zgodovine glasbe. najprej zadene ob vprašanje, kaj so to dejstva glasbene zgodovine. Starejša, "normalna" historiografska metoda se je osredotočala predvsem na predstavitev skladateljskih biografij, medtem ko sodobnejša bolj zbira, evidentira in ureja dokumente ter sezname del, čeprav gre v resnici zgolj za zgodovinske vire. Razjasnitev tega problema je tesno povezana s problematiko predmeta zgodovine glasbe, ki je zelo izmuzljiv, saj glasba obstaja zgolj v sedanjosti, v kateri izzveneva, torej v trenutnem posedanjanju. Zato je treba jasno razločevati med kulturami z notacijsko tradicijo in tistimi brez nje. Šele 
fiksacija glasbe z zapisom je omogočila stalno obnavljanje starejših del, kar lahko razumemo kot začetek glasbene zgodovine. Umetniško glasbeno delo tako obstaja v treh modusih: (1) v notnem tekstu je fiksirano nespremenljivo, (2) zveni vsakič nekoliko drugače, (3) obstaja pa tudi v našem glasbenem spominu v obliki vtisa. Zato je pomembno ločevati med "ustno" in "pisno" glasbo: prva je težko predmet glasbene zgodovine, saj obstaja v izročilu zgolj v danem trenutku samem. Podobno velja tudi za neumetniško glasbo, ki tudi ne prerašča v zgodovinsko danost. Ob pisanju zgodovine glasbe je pomembno še vprašanje, ali je zgodovinski lok smiselno opazovati skozi optiko stalnega napredka. Sledi svarilo, da je za tak pogled ponavadi krivo dejstvo, da o glasbi močno preteklih zgodovinskih obdobij niti nimamo dovolj podatkov. Poseben primer predstavlja tudi periodizacija glasbene zgodovine, ki pa ima v luči vprašanja, kaj je to glasba, šele sekundarni pomen. 